\title{
SEMIGROUP VARIETIES CLOSED FOR THE BRUCK EXTENSION
}

\author{
by FRANCIS PASTIJN and XIAOYING YAN
}

(Received 2 March, 1993)

1. Introduction. We shall show that there exists a chain, order isomorphic to the chain of real numbers, of semigroup varieties closed for the Bruck extension. The least semigroup variety closed for the Bruck extension will be obtained as the union of varieties in an infinite chain of semigroup varieties.

For any semigroup $S$, let $S^{1}$ be the semigroup $S$ if $S$ has an identity element and otherwise, $S^{1}$ will be the semigroup $S$ with an identity element adjoined. The set of natural numbers will be denoted by $\mathbb{N}$. On $\mathbb{N} \times S^{1} \times \mathbb{N}$ we can define a multiplication by putting

$$
(m, s, n)(p, t, q)=\left\{\begin{array}{lll}
(m, s, q+n-p) & \text { if } & n>p \\
(m, s t, q) & \text { if } & n=p \\
(m+p-n, t, q) & \text { if } & p>n
\end{array}\right.
$$

We thus obtain a semigroup which we denote by $B(S)$, and which is called the Bruck extension of $S$. It is easy to see that

$$
S \rightarrow B(S), \quad s \rightarrow(0, s, 0)
$$

is an embedding and that $B(S)$ is simple (see e.g. $\S 8.5$ of [3]).

A subvariety $\mathbf{V}$ of the variety $\mathbf{S}$ of all semigroups is closed for the Bruck extension if for every $S \in \mathbf{V}$ we have that $B(S) \in \mathbf{V}$. In particular, if $\mathbf{V}$ is closed for the Bruck extension, then each $S \in \mathbf{V}$ can be embedded in a simple semigroup of $\mathbf{V}$. Let $L(\mathbf{S})$ be the lattice of all semigroup varieties and $B L(\mathbf{S})$ the set of all varieties closed for the Bruck extension. In [5] it is shown that $B L(\mathbf{S})$ is a complete sublattice of $L(\mathbf{S})$. In this paper we shall further investigate $B L(\mathbf{S})$ and the way $B L(\mathbf{S})$ is embedded in $L(\mathbf{S})$. We shall show that $B L(\mathbf{S})$ contains a chain which is isomorphic to the chain of real numbers. Moreover, if $\mathbf{V} \in B L(\mathbf{S})$ and $\mathbf{V} \neq \mathbf{S}$, then we shall show that there exists an infinite ascending chain in $B L(\mathbf{S})$ with least element $\mathbf{V}$.

Since $B L(\mathbf{S})$ is a complete sublattice of $L(\mathbf{S})$ and $\mathbf{S} \in B L(\mathbf{S})$, it follows that for every $\mathbf{V} \in L(\mathbf{S})$ there exists a smallest semigroup variety $B \mathbf{V}$ which contains $\mathbf{V}$ and which is closed for the Bruck extension. It is easy to see that $B: L(\mathbf{S}) \rightarrow L(\mathbf{S}), \mathbf{B} \rightarrow B \mathbf{V}$ is a closure mapping (in the sense of [2]) and therefore $B$ is a complete $\vee$-homomorphism of $L(\mathbf{S})$ onto $B L(\mathbf{S})$. If $S$ is any semigroup, then define $B^{n}(S), n \in \mathbb{N}$, inductively by

$$
B^{0}(S)=S, \quad B^{n+1}(S)=B\left(B^{n}(S)\right) \text {. }
$$

For any $n \geq 0$, let $\varphi_{n}$ be the embedding

$$
\varphi_{n}: B^{n}(S) \rightarrow B^{n+1}(S), \quad a \rightarrow(0, a, 0)
$$

and let $B^{\omega}(S)$ be the direct limit of the directed family $\left(B^{n}(S), \varphi_{n}\right)_{n \in \mathbb{N}}$. Thus, $B^{\omega}(S)=\left(\bigcup_{n \in N} B^{n}(S)\right) / \theta$ where $\theta$ is the equivalence relation generated by the set of 
pairs $\left(a, a \varphi_{n}\right), n \in \mathbb{N}, a \in B^{n}(S)$. Then a result of [5] states that, given the variety $\operatorname{Var} S$ generated by the semigroup $S$, then $B \operatorname{Var} S=\operatorname{Var} B^{\omega}(S)=\bigcup_{n \in \mathbb{N}} \operatorname{Var} B^{n}(S)$. Clearly

$$
\operatorname{Var} S \subseteq \operatorname{Var} B(S) \subseteq \operatorname{Var} B^{2}(S) \subseteq \ldots \subseteq \operatorname{Var} B^{n}(S) \subseteq \ldots \subseteq B \operatorname{Var} S .
$$

We shall show that in general these inclusions are strict. In particular, if $T$ is a one-element semigroup and $\mathbf{T}=\operatorname{Var} T$ is the trivial variety, then $B \mathbf{T}$ is the least element of $B L(\mathbf{S})$ and the inclusions in

$$
\mathbf{T} \subset \operatorname{Var} B(T) \subset \operatorname{Var} B^{2}(T) \subset \ldots \subset \operatorname{Var} B^{n}(T) \subset \ldots \subset B \mathbf{T}
$$

will be shown to be strict. Here $B^{n}(T)$ is the Munn semigroup (in the sense of [6]) of a dually well-ordered chain of order type $\left(\omega^{n}\right)^{*}$ and $B \mathbf{T}=\operatorname{Var} B^{\omega}(T)$, where $B^{\omega}(T)$ is the Munn semigroup of a dually well-ordered chain of order type $\left(\omega^{\omega}\right)^{*}$. Therefore each of these semigroups is bisimple (see also $\S 8.5$ of [3]). The semigroup $B(T)$ is also called the bicyclic semigroup and the variety generated by $B(T)$ has been studied in several papers: we refer to [4] for further references.

2. Chains in $B L(\mathbf{S})$. As in [5] we denote the elements of the bicyclic semigroup $B(T)$ by $(m, n), m, n \in \mathbb{N}$, and we introduce the relations $\sqsubset$ and $\sqsupset$ on $B(T)$ by

$$
\begin{aligned}
& (m, n) \sqsupset(p, q) \Leftrightarrow n>p, \\
& (m, n) \sqsubset(p, q) \Leftrightarrow n<p .
\end{aligned}
$$

We let $X$ be a countably infinite set of variables and $X^{*}\left[X^{+}\right]$be the set of [nonempty] words over the alphabet $X$. The equality on $X^{+}$will be denoted by $\equiv$.

The following useful result was proved in [5].

Result 1. Let $u=u\left(x_{1}, x_{2}\right)$ and $v=v\left(x_{1}, x_{2}\right)$ be words involving the two variables $x_{1}$ and $x_{2}$ only. If for $\hat{a}=(m, n)$ and $\hat{b}=(p, q)$ of $B(T)$ we have

$$
u(\hat{a}, \hat{b}) \sqsupset \hat{a} \hat{b} \quad \text { and } \quad u(\hat{a}, \hat{b}) \sqsupset \hat{b} \hat{a}
$$

or

$$
\hat{a} \hat{b} \sqsubset v(\hat{a}, \hat{b}) \text { and } \hat{b} \hat{a} \sqsubset v(\hat{a}, \hat{b}) \text {, }
$$

then for any semigroup $S, s, t \in S^{1}$, and $\bar{a}=(m, s, n)$ and $\bar{b}=(p, t, q)$ in $B(S)$, we have that

$$
u(\bar{a}, \bar{b}) \bar{a} \bar{b} v(\bar{a}, \bar{b})=u(\bar{a}, \bar{b}) \bar{b} \bar{a} v(\bar{a}, \bar{b}) .
$$

For any $k, l \geq 2$ we put for $x_{1}, x_{2} \in X$,

$$
\begin{aligned}
& a_{k, l} \equiv x_{1} x_{2}^{k} x_{1} x_{1} x_{2} x_{1} x_{2}^{\prime} x_{1}, \\
& b_{k, l} \equiv x_{1} x_{2}^{k} x_{1} x_{2} x_{1} x_{1} x_{2}^{\prime} x_{1},
\end{aligned}
$$

and for $u, v \in X^{+}$we then have

$$
\begin{aligned}
& a_{k, l}(u, v) \equiv u v^{k} u u v u v^{\prime} u, \\
& b_{k, l}(u, v) \equiv u v^{k} u v u u v^{\prime} u .
\end{aligned}
$$

The following lemma will be crucial for the proof of the main result in this section. 
LEMMA 2. Let $S$ be any semigroup, let $k, l \geq 3$ and $u, v \in X^{+}$. In an evaluation, where the variables which occur in $u$ or $v$ are replaced by elements of $B(S)$, let $\bar{u}=(m, s, n)$ and $\bar{v}=(p, t, q)$ be the elements of $B(S)$ which correspond to $u$ and $v$ respectively. Then, if $m=n=p=q$ does not hold, $a_{k . l}(\bar{u}, \bar{v})=b_{k . l}(\bar{u}, \bar{v})$ is a true equality in $B(S)$.

Proof. We consider the elements $\hat{\imath}=(m, n)$ and $\hat{v}=(p, q)$ of the bicyclic semigroup $B(T)$ which correspond to $\bar{u}$ and $\bar{v}$, respectively. There are numerous cases to be considered. Each of these cases then makes use of Result 1.

It suffices to show that for each case we have either

$$
\hat{a} \hat{v} \sqsubset \hat{u} \hat{v}^{\prime} \hat{u}, \quad \hat{v} \hat{u} \sqsubset \hat{u} \hat{v}^{\prime} \hat{u},
$$

or

$$
\hat{u} \hat{v}^{k} \hat{u} \sqsupset \hat{u} \hat{v}, \quad \hat{u} \hat{v}^{k} \hat{u} \sqsupset \hat{v} \hat{u} .
$$

We now give a survey of all the cases and indicate which one of (4) or (5) applies:

$$
\begin{aligned}
& \text { (i) } m>n, p<q, n>p, m-n>q-p:(4), \\
& \text { (ii) } m>n, p<q, n>p, m-n \leq q-p:(5), \\
& \text { (iii) } m>n, p<q, n \leq p, m-n>q-p:(4), \\
& \text { (iv) } m>n, p<q, n \leq p, m-n \leq q-p:(5), \\
& \text { (v) } n<m \leq p=q:(4), \\
& \text { (vi) } n \leq p=q<m:(4), \\
& \text { (vii) } p=q<n<m:(4), \\
& \text { (viii) } m>n, p>q, p>n, q>m:(4), \\
& \text { (ix) } m>n, p>q, p>n, q \leq m:(4), \\
& \text { (x) } m>n, p>q, p \leq n:(4), \\
& \text { (xi) } q<p \leq m=n:(4), \\
& \text { (xii) } q \leq m=n<p:(4), \\
& \text { (xiii) } m=n<q<p:(4), \\
& \text { (xiv) } p<q \leq m=n:(5), \\
& \text { (xv) } p \leq m=n<q:(5), \\
& \text { (xvi) } m=n<p<q:(5), \\
& \text { (xvii) } m<n, p>q, n>p, n-m \leq p-q:(4), \\
& \text { (xviii) } m<n, p>q, n>p, n-m>p-q:(5), \\
& \text { (xix) } m<n, p>q, n \leq p, m-n \geq q-p:(4), \\
& \text { (xx) } m<n, p>q, n \leq p, m-n<q-p:(5), \\
& \text { (xxi) } m<n \leq p=q:(5), \\
& \text { (xxii) } m \leq p=q<n:(5), \\
& \text { (xxiii) } p=q<m<n:(5), \\
& \text { (xxiv) } m<n, p<q, q<m:(5), \\
& \text { (xxv) } m<n, p<q, q \geqslant m, p<n:(5), \\
& \text { (xxvi) } m<n, p<q, q \geq m, p \geq n:(5) . \\
&
\end{aligned}
$$

Only one case has been left out in the above, namely the case

(xxvii) $m=n, p=q$,

which can be treated as follows. If $m=n>p=q$, then $a_{k, l}(\bar{u}, \bar{v})=\left(m, s^{5}, m\right)=b_{k, l}(\bar{u}, \bar{v})$ and if $m=n<p=q$, then $a_{k, l}(\bar{u}, \bar{v})=\left(p, t^{k+1+1}, q\right)=b_{k, l}(\bar{u}, \bar{v})$. 
The proof for each of the above cases follows from a routine computation. By way of example we treat the case (xix), where $m<n, p>q, n \leq p, m-n \geq q-p$.

If $q \geq m$, then

$$
\begin{aligned}
\hat{u} \hat{v} & =(m+p-n, q), \quad \hat{v} \hat{u}=(p, n+q-m), \\
\hat{u}^{\prime} \hat{v} & =(m+l p-(l-1) q-n, n+q-m),
\end{aligned}
$$

and since

$$
\begin{gathered}
q<q+l(p-q)-(n-m)=m+l p-(l-1) q-n, \\
n+q-m<n+q-m+l(p-q)-(l-1)(n-m)=m+l p-(l-1) q-n,
\end{gathered}
$$

we have $\hat{u} \hat{v} \sqsubset \hat{u} \hat{v}^{\prime} \hat{u}, \hat{v} \hat{u} \sqsubset \hat{u} \hat{v}^{\prime} \hat{u}$ and $a_{k, l}(\bar{u}, \bar{v})=b_{k, l}(\bar{u}, \bar{v})$ by Result 1 . If $q<m$, then

where

$$
\begin{gathered}
\hat{u} \hat{v}=(m+p-n, q), \quad \hat{v} \hat{u}=(p+m-q, n), \\
\hat{u} \hat{v}^{\prime} \hat{u}=(2 m+l p-l q-n, n),
\end{gathered}
$$

$$
\begin{gathered}
q<m<m+l(p-q)-(n-m)=2 m+l p-l q-n, \\
n<n+l(p-q)-2(n-m)=2 m+l p-l q-n,
\end{gathered}
$$

thus $\hat{u} \hat{v} \sqsubset \hat{u} \hat{v}^{\prime} \hat{u}, \hat{v} \hat{u} \sqsubset \hat{u} \hat{v}^{\prime} \hat{u}$, and so $a_{k, l}(\bar{u}, \bar{v})=b_{k, l}(\bar{u}, \bar{v})$ by Result 1 .

The following theorem generalizes a result of [5].

THEOREM 3. For any semigroup $S$ and $k, l \geq 3, e, f \geq 1$, the identity $a_{k, l}\left(x_{1}^{e}, x_{2}^{f}\right)=$ $b_{k, l}\left(x_{1}^{e}, x_{2}^{f}\right)$ holds in $S$ if and only if this identity holds in $B(S)$.

Proof. Clearly, if $a_{k, l}\left(x_{1}^{e}, x_{2}^{f}\right)=b_{k, l}\left(x_{1}^{e}, x_{2}^{f}\right)$ holds in $B(S)$, then this identity holds in $S$, since $S$ is a subsemigroup of $B(S)$. Conversely, let us assume that $a_{k, l}\left(x_{1}^{e}, x_{2}^{f}\right)=b_{k, l}\left(x_{1}^{e}, x_{2}^{f}\right)$ holds in $S$ and let $\bar{u}=(m, s, n), \bar{v}=(p, t, q)$ be the elements of $B(S)$ which result from an evaluation where $u \equiv x_{1}^{e}$ and $v \equiv x_{2}^{f}$, respectively. We must show that $a_{k, l}(\bar{u}, \bar{v})=b_{k, l}(\bar{u}, \bar{v})$ holds in $B(S)$. By the preceding lemma, this equality holds unless maybe $m=n=p=q$. Let us look at the case where $m=n=p=q$. If $x_{1}$ is replaced by $a \in B(S)$, then $\bar{u}=a^{e}=(m, s, m)$, whence $a=(m, g, m)$ and $s=g^{e}$ for some $g \in S$. Similarly, $x_{2}$ is replaced by $b \in B(S)$, where $b=(m, h, m)$ and $t=h^{f}$ for some $h \in S$. Therefore

$$
\begin{aligned}
a_{k, l}(\bar{u}, \bar{v}) & =\left(m, a_{k, l}\left(g^{e}, h^{f}\right), m\right) \\
& =\left(m, b_{k, l}\left(g^{e}, h^{f}\right), m\right) \\
& =b_{k, l}(\bar{u}, \bar{v}) .
\end{aligned}
$$

Therefore $a_{k, l}\left(x_{1}^{e}, x_{2}^{f}\right)=b_{k, l}\left(x_{1}^{e}, x_{2}^{f}\right)$ holds in $B(S)$.

In [5], it was already noted that the identities of the statement of Theorem 3 hold in $B^{\omega}(T)$; therefore the least element $B \mathbf{T}=\operatorname{Var} B^{\omega}(T)$ of the lattice $B L(\mathbf{S})$ is certainly properly contained in $\mathbf{S}$.

For any $A \subseteq\{(k, l, e, f) \mid k, l \geq 3, e, f \geq 1\}$ we let $\mathbf{V}_{A}$ be the variety determined by the identities of the form $a_{k, l}\left(x_{1}^{e}, x_{2}^{f}\right)=b_{k, l}\left(x_{1}^{e}, x_{2}^{f}\right),(k, l, e, f) \in A$.

Corollary 4. For any $A \subseteq\{(k, l, e, f) \mid k, l \geq 3, e, f \geq 1\}, \mathbf{v}_{A}$ belongs to $B L(\mathbf{S})$.

As in [7] the order type of the chain of real numbers is denoted by $\lambda$. Then $\lambda$ is also 
the order type of the chain of real numbers ]0,1[. Each real number $0<n<1$ has a unique nonterminating decimal representation in which there is no infinite succession of zeros; similarly, every real number $0<n<1$ can be written in a unique way as $n=\sum_{k=1}^{\infty} i_{k} 2^{-k}$ with $i_{k}=0$ or $i_{k}=1$ for all $k \geq 1$ and such that for every $l \geq 1$ there exists $j>l$ with $i_{j}=1$. With this notation we put, for every $j \geq 1, l(n, j)=2^{j-1} i_{1}+2^{j-2} i_{2}+\ldots+$ $2^{\prime \prime} i_{j}+1$. Clearly, if $0<n, n^{\prime}<1$, then $n<n^{\prime}$ if and only if $l(n, k)<l\left(n^{\prime}, k\right)$ for some $k \geq 1$, and if this is the case, then $l(n, j)<l\left(n^{\prime}, j\right)$ for all $j \geq k$.

THEOREM 5. $B L(\mathbf{S})$ contains a chain of order type $\lambda$.

Proof. Let $p_{1}, p_{2}, \ldots$ be the infinite strictly increasing sequence of prime numbers, with $p_{1} \geq 5$. For a real number $0<n<1$, let $A_{n}=\left\{\left(3,3,1,2^{l(n, j)} p_{j} \mid j \geq 1\right\}\right.$. We shall show that the mapping

$$
\varphi:] 0,1\left[\rightarrow B L(\mathbf{S}), \quad n \rightarrow \mathbf{V}_{A_{n}}\right.
$$

is an order isomorphism of ]0,1[ onto a subchain of $B L(\mathbf{S})$. Clearly, by Corollary 4 , $\mathbf{V}_{A_{n}} \in B L(\mathbf{S})$ for every $0<n<1$.

Let $n \leq n^{\prime}$. Then $l(n, j) \leq l\left(n^{\prime}, j\right)$ for every $j \geq 1$. The variety $\mathbf{V}_{A_{n}}$ is determined by the identities of the form

$$
a_{3,3}\left(x_{1}, x_{2}^{2^{(n n j)} p_{j}}=b_{3,3}\left(x_{1}, x_{2}^{2^{(n, n j} p_{j}}\right), \quad j \geq 1,\right.
$$

whereas $\mathbf{V}_{A_{n}}$ is determined by the identities of the form

$$
a_{3,3}\left(x_{1}, x_{2}^{\left.2^{\left(n n^{\prime} j\right.}\right) p_{j}}\right)=b_{3,3}\left(x_{1}, x_{2}^{2\left(n^{\prime} j\right)} p_{j}\right), \quad j \geq 1 .
$$

If for some $j \geq 1$ we have $l(n, j)<l\left(n^{\prime}, j\right)$, then the substitution in (7) of $x_{2}$ by $x_{2}^{2^{l\left(n^{\prime} j\right)-\left(n n^{\prime}\right)}}$ yields the identity (8). Therefore, if $n \leq n^{\prime}$ then the identities in (8) follow from the corresponding identities (7), so $\mathbf{V}_{\boldsymbol{A}_{n}} \subseteq \mathbf{V}_{\boldsymbol{A}_{n}}$. Therefore the mapping of (6) is order preserving.

We next show that $\varphi$ is a injection. Let us suppose that $n<n^{\prime}$. Then for some $k \geq 1$, $l(n, k)<l\left(n^{\prime}, k\right)$. We show that the identity

$$
a_{3,3}\left(x_{1}, x_{2}^{2^{(n, k)} p_{k}}\right)=b_{3,3}\left(x_{1}, x_{2}^{2^{(n, k)} p_{k}}\right)
$$

does not follow from the defining identities (8) for $\mathbf{V}_{A_{n}}$. Here

$$
a_{3,3}\left(x_{1}, x_{2}^{2^{2(n, k)} p_{k}}\right) \equiv x_{1} 2_{2}^{2(n, k) 3 p_{k}} x_{1} x_{1} x_{2}^{2^{(n+k)} p_{k}} x_{1} x_{2}^{2^{(n+. k)} 3 p_{k} x_{1}}
$$

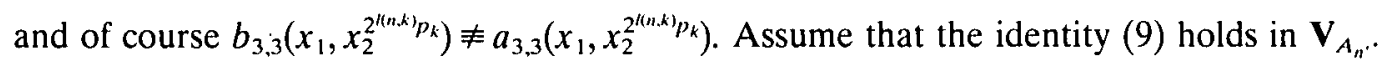
Then there exists $l>1, u_{1}, \ldots, u_{l}, v_{1}, \ldots, v_{l}, y_{1}, \ldots, y_{l}, z_{1}, \ldots, z_{l} \in X^{*}$ such that

$$
\begin{array}{cl}
a_{3,3}\left(x_{1}, x_{2}^{2(n, k)} p_{k}\right) \equiv y_{1} u_{1} z_{1}, & y_{1} v z_{l} \equiv b_{3,3}\left(x_{1}, x_{2}^{2^{2(n, k)} p_{k}}\right), \\
y_{i} v_{i} z_{i} \equiv y_{i+1} u_{i+1} z_{i+1} & \text { for all } 1 \leq i \leq l,
\end{array}
$$

and such that for all $i, u_{i} \not \equiv v_{i}$ and $u_{i}=v_{i}$ is a substitution instance of one of the defining identities (8) of $\mathbf{V}_{A_{n} \text {. }}$. In particular,

$$
u_{1} \equiv a_{3,3}\left(a_{1}, a_{2}^{2\left(n^{\prime} j^{\prime}\right) p_{j}}\right) \quad \text { or } \quad u_{1} \equiv b_{3,3}\left(a_{1}, a_{2}^{\left.2^{\left(n^{\prime} j\right)} p_{i}\right)}\right.
$$

for some $a_{1}, a_{2} \in\left\{x_{1}, x_{2}\right\}^{+}$. If $x_{1}$ occurs in $a_{2}$, then, since $l\left(n^{\prime}, j\right) \geq 1$ and $p_{j} \geq 5, x_{1}$ occurs 
more than 5 times in $u_{1}$, which is impossible since $x_{1}$ occurs precisely 5 times in $y_{1} u_{1} z_{1}$. Thus $a_{2} \equiv x_{2}^{s}$ for some $s \geq 1$. If $x_{1}$ does not occur in $a_{1}$, then $a_{1} \equiv x_{2}^{t}$ for some $t \geq 1$ and $u_{1} \equiv v_{1}$, which is contrary to $u_{i} \not \equiv v_{i}$ for all $i$. Thus $x_{1}$ occurs in $a_{1}$. Since there are precisely 5 disjoint occurrences of $a_{1}$ as a subword in $u_{1}$, and precisely 5 occurrences of $x_{1}$ in $y_{1} u_{1} z_{1}$, we have that $x_{1}$ occurs precisely once in $a_{1}$. Further, since $x_{1}$ is the first and the last letter of $y_{1} u_{1} z_{1}$ we have $a_{1} \equiv x_{1}$, whereas $y_{1}$ and $z_{1}$ are empty. Thus $a_{3.3}\left(x_{1}, x_{2}^{2^{(n+k)} p_{k}}\right) \equiv u_{1} \equiv a_{3,3}\left(x_{1}, x_{2}^{\left.s 2^{\left(n n^{\prime} \cdot j\right)} p_{i}\right)}\right.$, whence $2^{\prime(n, k)} p_{k}=s 2^{\prime\left(n^{\prime} j\right)} p_{j}$. Since $p_{j}$ and $p_{k}$ are prime numbers, both greater than 2 , we have $p_{k}=p_{j}$, thus $k=j$. Therefore $l(n, k) \geq$ $l\left(n^{\prime}, k\right)$, a contradiction. Hence the identity (9) holds in $\mathbf{V}_{A_{n}}$ but not in $\mathbf{V}_{A_{n}}$. We conclude that $\mathbf{V}_{A_{n}}$ is strictly contained in $\mathbf{V}_{A_{n}}$, and the mapping $\varphi$ is an order isomorphism of ]0,1[ onto a subchain of $B L(\mathbf{S})$.

In [5] we showed the following

REsult 6. If $\mathbf{V}$ is a semigroup variety which is closed for the Bruck extension, and $\mathbf{V}$ is not the variety $\mathbf{S}$ of all semigroups, then $\mathbf{V}$ is contained in a semigroup variety which is not closed for the Bruck extension.

The following result is interesting in its own right. For any $u \in X^{+}, l(u)$ will denote the length of $u$, i.e. the number of letters from $X$ which occur in $u$.

\section{THEOREM 7. If $\mathbf{V}$ is a proper subvariety of $\mathbf{S}$, then $B \mathbf{V}$ is a proper subvariety of $\mathbf{S}$.}

Proof. Since $\mathbf{V}$ is a proper subvariety of $\mathbf{S}$ there exists a nontrivial identity $u=v$ in two variables which is satisfied in $\mathbf{V}$ (see Theorem 9.10 of [3]). If $l(u) \neq l(v)$, then $x^{\prime(u)}=x^{\prime(v)}$ is satisfied in $\mathrm{V}$, thus with $u^{\prime} \equiv x_{1}^{\prime(u)} x_{2}^{\prime(v)}$ and $v^{\prime} \equiv x_{1}^{\prime(v)} x_{2}^{\prime(u)}, u^{\prime}=v^{\prime}$ is a nontrivial identity in two variables which is satisfied in $\mathbf{V}$ and such that $l\left(u^{\prime}\right)=l\left(v^{\prime}\right)$. Therefore, without loss of generality, we can assume that there exists a nontrivial identity, $u=v$ in the two variables $x_{1}, x_{2}$, which is satisfied in $\mathbf{V}$ and such that $l(u)=l(v)$.

Clearly, since $u=v$ is a nontrivial identity, so is $u\left(a_{3,3}, b_{3,3}\right)=v\left(a_{3,3}, b_{3,3}\right)$. We show by induction on $k \geq 0$, that this identity is satisfied in $B^{k}(S)$ for $S \in \mathbf{V}$, where $B^{0}(S)=S$. Of course this statement holds if $k=0$. Assume that the identity $u\left(a_{3,3}, b_{3,3}\right)=v\left(a_{3,3}, b_{3,3}\right)$ is satisfied in $B^{k}(S)$ for every $S \in \mathbf{V}$ and $k \geq 0$. Let us evaluate the two variables $x_{1}$ and $x_{2}$ which occur in this identity by the elements $a=(m, s, n), b=(p, t, q) \in B^{k+1}(S)$, respectively, where $s, t \in B^{k}(S), S \in \mathbf{V}$. If $m=n=p=q$ does not hold, then by Lemma 2, $a_{3,3}(a, b)=b_{3,3}(a, b)$ holds in $B^{k+1}(S)$ and since $l(u)=l(v), u\left(a_{3,3}(a, b), b_{3,3}(a, b)\right)=$ $v\left(a_{3,3}(a, b), b_{3,3}(a, b)\right)$ holds in $B^{k+1}(S)$. Otherwise, $m=n=p=q$ and using the induction hypothesis

$$
\begin{aligned}
u\left(a_{3,3}(a, b), b_{3,3}(a, b)\right) & =\left(m, u\left(a_{3,3}(s, t), b_{3,3}(s, t)\right), m\right) \\
& =\left(m, v\left(a_{3,3}(s, t), b_{3,3}(s, t)\right), m\right) \\
& =v\left(a_{3,3}(a, b), b_{3,3}(a, b)\right) .
\end{aligned}
$$

Therefore the identity $u\left(a_{3,3}, b_{3,3}\right)=v\left(a_{3,3}, b_{3,3}\right)$ is satisfied in $B^{k+1}(S)$ for every $S \in \mathbf{V}$. Using induction and the fact that for every $S \in \mathbf{V}, B^{\omega}(S)$ is the direct limit of the $B^{n}(S)$, $n \geq 0$, we then have that this identity is satisfied by $B^{\omega}(S)$ for every $S \in \mathbf{V}$. In particular, if $S$ is free on $X$ in $\mathbf{V}$, then $\mathbf{V}=\operatorname{Var} S$ and $B \mathbf{V}=\operatorname{Var} B^{\omega}(S)$. Therefore $u\left(a_{3,3}, b_{3,3}\right)=$ $v\left(a_{3,3}, b_{3,3}\right)$ is a nontrivial identity satisfied in $B \mathbf{V}$. This implies that $B \mathbf{V}$ is a variety which is properly contained in $\mathbf{S}$. 
Result 6 and Theorem 7 thus imply

THEOREM 8. If $\mathbf{V} \neq \mathbf{S}$ and $\mathbf{V} \in B L(\mathbf{S})$, then in $B L(\mathbf{S})$ there exists an infinite chain with least element $\mathbf{V}$.

3. Subvarieties of $B \mathbf{T}$. The variety $B \mathbf{T}=\operatorname{Var} B^{\omega}(T)$ is the least element of $B L(\mathbf{S})$. Therefore, if $\mathbf{V} \subseteq \mathbf{V a r} B^{\omega}$, then $B \mathbf{V}=B \mathbf{T}$. Thus for instance, since the variety $\mathbf{B}$ of bands is contained in the variety generated by the bicyclic semigroup $B(T)$ [4], we have $B \mathbf{B}=B \mathbf{T}$. From [5] we have that $\operatorname{Var} B(T)$ is properly contained in $B \mathbf{T}$. We shall show that $\operatorname{Var} B^{\prime \prime}(T)$ is properly contained in $B \mathbf{T}$ for each $n \in \mathbb{N}$. In [5], we already showed that $a_{2,2}=b_{2,2}$ is satisfied in $B(T)$ but not in $B^{2}(T)$. Therefore the variety generated by the bicyclic semigroup $B(T)$ is certainly properly contained in the least element $B \mathbf{T}$ of $B L(\mathbf{S})$. We first have

LeMma 9. Let $\hat{a}=(m, n)$ and $\hat{b}=(p, q)$ be such that either both $n-m$ and $q-p$ are positive, or both are negative. Then for every semigroup $S, s, t \in S^{1}$ and $\bar{a}=(m, s, n)$, $\bar{b}=(p, t, q)$ in $B(S)$ we have that $a_{2,2}(\bar{a}, \bar{b})=b_{2,2}(\bar{a}, \bar{b})$.

Proof. We shall assume that $n-m$ and $q-p$ are both positive. The case where both $n-m$ and $q-p$ are negative can be treated in a similar way. In view of Result 1 , it suffices to show that $\hat{a} \hat{b}^{2} \hat{a} \sqsupset \hat{a} \hat{b}$ and $\hat{a} \hat{b}^{2} \hat{a} \sqsupset \hat{b} \hat{a}$. We can consider two cases.

Case 1. $p \geq n$. Then $q>p \geq n>m$ and $\hat{a} \hat{b}=(m+p-n, q), \hat{b} \hat{a}=(p, n+q-m)$, $\hat{a} \hat{b}^{2} \hat{a}=(m+p-n, n+2 q-p-m)$. Since

$$
n+2 q-p-m>2 q-p>p>m+p-n
$$

we have $\hat{a} \hat{b}^{2} \hat{a} \sqsupset \hat{a} \hat{b}$ and $\hat{a} \hat{b}^{2} \hat{a} \sqsupset \hat{b} \hat{a}$.

Case 2. $p<n$. Then $\hat{a} \hat{b}=(m, q+n-p)$ and $\hat{a} \hat{b}^{2} \hat{a}=(f, n+e)$ for some $e, f \geq 0$. If $q \leq m$, then $\hat{b} \hat{a}=(p+m-q, n)$ where $p+m-q<m<n$. If $q>m$, then $\hat{b} \hat{a}=(p, n+$ $m-q$ ). Thus again $\hat{a} \hat{b}^{2} \hat{a} \sqsupset \hat{a} \hat{b}$ and $\hat{a} \hat{b}^{2} \hat{a} \sqsupset \hat{b} \hat{a}$.

The main theorem of this section is then as follows.

THEOREM 10. For any $n \geq 1$, $\operatorname{Var} B^{n}(T)$ is a proper subvariety of $\operatorname{Var} B^{n+1}(T)$.

Proof. The above statement follows from the above mentioned result from [5] for the case $n=1$. For $n \geq 2$ we shall now construct an identity $u_{n}=v_{n}$ which is satisfied in $B^{\prime \prime}(T)$ but not in $B^{n+1}(T)$. We put for a given $n \geq 2$,

$$
\begin{aligned}
w_{n, 1}^{(n)} & \equiv x_{1} x_{2} \ldots x_{n} x_{n+1}, \\
w_{n, 2}^{(n)} & \equiv x_{2} x_{1} x_{3} \ldots x_{n} x_{n+1}, \\
\vdots & \\
w_{n, n}^{(n)} & \equiv x_{n} x_{1} x_{2} \ldots x_{n-1} x_{n+1}, \\
w_{n-1,1}^{(n)} & \equiv w_{n, 1}^{(n)} \ldots w_{n, n}^{(n)}, \\
w_{n-1,2}^{(n)} & \equiv w_{n, 2}^{(n)} w_{n, 1}^{(n)} w_{n, 3}^{(n)} \ldots w_{n, n}^{(n)}, \\
\vdots & \\
w_{n-1, n-1}^{(n)} & \equiv w_{n, n-1}^{(n)} w_{n, 1}^{(n)} \ldots w_{n, n-2}^{(n)} w_{n, n}^{(n)},
\end{aligned}
$$


and inductively, for $2 \leq k \leq n$,

$$
\begin{aligned}
w_{k, 1}^{(n)} & \equiv w_{k+1,1}^{(n)} \ldots w_{k+1, k+1}^{(n)} \\
w_{k, 2}^{(n)} & \equiv w_{k+1,2}^{(n)} w_{k+1,1}^{(n)} w_{k+1,3}^{(n)} \ldots w_{k+1, k+1}^{(n)} \\
& \vdots \\
w_{k, k}^{(n)} & \equiv w_{k+1, k}^{(n)} w_{k+1,1}^{(n)} \ldots w_{k+1, k-1}^{(n)} w_{k+1, k+1}^{(n)} .
\end{aligned}
$$

Thus

Finally,

$$
w_{2,1}^{(n)} \equiv w_{3,1}^{(n)} w_{3,2}^{(n)} w_{3,3}^{(n)}, \quad w_{2,2}^{(n)} \equiv w_{3,2}^{(n)} w_{3,1}^{(n)} w_{3,3}^{(n)}
$$

$$
u_{n} \equiv a_{2,2}\left(w_{2,1}^{(n)}, w_{2,2}^{(n)}\right), \quad v_{n} \equiv b_{2,2}\left(w_{2,1}^{(n)}, w_{2,2}^{(n)}\right) .
$$

We prove by induction that for all $n \geq 2, u_{n}=v_{n}$ is satisfied in $B^{n}(T)$ but not in $B^{n+1}(T)$. We first consider the case $n=2$. Then $w_{2,1}^{(2)} \equiv x_{1} x_{2} x_{3}, w_{2,2}^{(2)} \equiv x_{2} x_{1} x_{3}$. Let $a_{1}, a_{2}, a_{3}$ be any elements of $B^{2}(T), \bar{a}=a_{1} a_{2} a_{3}=(m, s, n)$ and $\bar{b}=a_{2} a_{1} a_{3}=(p, t, q)$ for some $\hat{a}=(m, n), \hat{b}=(p, q), s, t \in B(T)$. Since

$$
\sigma: B^{2}(T) \rightarrow \mathbb{Z},(k, r, l) \rightarrow l-k
$$

is a homomorphism of $B^{2}(T)$ onto the additive group of the integers $\mathbb{Z}$, which is commutative,

$$
n-m=\bar{a} \sigma=\left(a_{1} a_{2} a_{3}\right) \sigma=\left(a_{2} a_{1} a_{3}\right) \sigma=\bar{b} \sigma=q-p .
$$

Then $n-m \neq 0$ if and only if $q-p \neq 0$ and in this case we can apply Lemma 9 to obtain $a_{2,2}(\bar{a}, \bar{b})=b_{2,2}(\bar{a}, \bar{b})$. Otherwise $m=n$ and $p=q$. Then if $m>p$, we have $a_{2,2}(\bar{a}, \bar{b})=$ $\left(m, s^{5}, m\right)=b_{2,2}(\bar{a}, \bar{b})$, whereas if $m<p$, then $a_{2,2}(\bar{a}, \bar{b})=\left(p, t^{5}, p\right)=b_{2,2}(\bar{a}, \bar{b})$. If $m=$ $n=n=p=q$, then $a_{2,2}(\bar{a}, \bar{b})=\left(m, a_{2,2}(s, t), m\right)=\left(m, b_{2,2}(s, t), m\right)=b_{2,2}(\bar{a}, \bar{b})$, since $a_{2,2}=b_{2,2}$ is satisfied in $B(T)$ by [1]. Therefore $u_{2}=v_{2}$ is satisfied in $B^{2}(T)$. Substitution for $x_{1}, x_{2}$ and $x_{3}$ by the elements

$$
\begin{aligned}
& b_{1}=(0,(0,(0,1), 1), 1), \\
& b_{2}=(0,(1,(0,1), 0), 1), \\
& b_{3}=(2,(0,(0,0), 0), 0),
\end{aligned}
$$

respectively, of $B^{3}(T)$ then gives

$$
\begin{gathered}
b_{1} b_{2} b_{3}=(0,(0,(0,1), 1), 0), \\
b_{2} b_{1} b_{3}=(0,(1,(0,1), 0), 0), \\
u_{2}\left(b_{1}, b_{2}, b_{3}\right)=a_{2,2}\left(b_{1} b_{2} b_{3}, b_{2} b_{1} b_{3}\right)=(0,(1,(0,4), 1), 0), \\
v_{2}\left(b_{1}, b_{2}, b_{3}\right)=b_{2,2}\left(b_{1} b_{2} b_{3}, b_{2} b_{1} b_{3}\right)=(0,(1,(0,6), 1), 0),
\end{gathered}
$$

and so $u_{2}=v_{2}$ is not satisfied in $B^{3}(T)$.

We shall now assume that $n \geq 2$ and that for all $2 \leq k \leq n, u_{k}=v_{k}$ is satisfied in $B^{k}(T)$ but not in $B^{k+1}(T)$. We shall show that $u_{n+1}=v_{n+1}$ is satisfied in $B^{n+1}(T)$ but not in $B^{n+2}(T)$. We shall evaluate the variables $x_{1}, \ldots, x_{n+2}$ which occur in $u_{n+1}=v_{n+1}$ by elements $a_{1}, \ldots, a_{n+2}$, respectively, of $B^{n+1}(T)$. The element of $B^{n+1}(T)$ which corresponds to $w_{j, l}^{(n+1)}$ in this evaluation we denote by $\bar{w}_{j, l}^{(n+1)}$ for each $j$ and $l$. Again

$$
\sigma: B^{n+1}(T) \rightarrow \mathbb{Z},(p, t, q) \rightarrow q-p
$$


is a homomorphism onto the additive group $\mathbb{Z}$, and so

$$
\bar{w}_{n+1,1}^{(n+1)} \sigma=\left(a_{1} \ldots a_{n+2}\right) \sigma=\left(a_{j} a_{1} a_{2} \ldots a_{n+2}\right) \sigma=\bar{w}_{n+1, j}^{(n+1)} \sigma
$$

for every $1 \leq j \leq n+1$. Using induction we can now easily show that

$$
\bar{w}_{2,1}^{(n+1)} \sigma=\bar{w}_{2,2}^{(n+1)} \sigma=\frac{(n+1) !}{2}\left(\bar{w}_{n+1,1}^{(n+1)} \sigma\right)
$$

Therefore, if $\bar{w}_{n+1,1}^{(n+1)} \sigma \neq 0$, then $a_{2,2}\left(\bar{w}_{2,1}^{(n+1)}, \bar{w}_{2,2}^{(n+1)}\right)=b_{2,2}\left(\bar{w}_{2,1}^{(n+1)}, \bar{w}_{2,2}^{(n+1)}\right)$ follows from Lemma 9. Thus $u_{n+1}\left(a_{1}, \ldots, a_{n+2}\right)=v_{n+1}\left(a_{1}, \ldots, a_{n+2}\right)$ in this case. We now consider the case where $\bar{w}_{n+1,1}^{(n+1)} \sigma=0$. Using (10) we can suppose that

$$
\bar{w}_{n+1,1}^{(n+1)}=\left(m_{1}, s_{1}, m_{1}\right), \ldots, \bar{w}_{n+1, n+1}^{(n+1)}=\left(m_{n+1}, s_{n+1}, m_{n+1}\right),
$$

with $s_{j} \in B^{n}(T)$ for $1 \leq j \leq n+1$. Then

$$
\bar{w}_{n j}^{(n+1)}=\left(m, w_{n j}^{(n)}\left(t_{1}, \ldots, t_{n+1}\right), m\right), \quad 1 \leq j \leq n,
$$

where $m=\max \left\{m_{1}, \ldots, m_{n+1}\right\}$ and $s_{l}=t_{l}$ if $m_{l}=m$ and $1=t_{l}$ if $m_{l}<m$. Using induction we can then easily show that

$$
\bar{w}_{2 j}^{(n+1)}=\left(m, w_{2, j}^{(n)}\left(t_{1}, \ldots, t_{n+1}\right), m\right) \quad j=1,2 .
$$

Using the induction hypothesis we then have that

$$
\begin{aligned}
u_{n+1}\left(a_{1}, \ldots, a_{n+2}\right) & =a_{2,2}\left(\bar{w}_{2,1}^{(n+1)}, \bar{w}_{2,2}^{(n+1)}\right) \\
& =\left(m, a_{2,2}\left(w_{2,1}^{(n)}\left(t_{1}, \ldots, t_{n+1}\right), w_{2,2}^{(n)}\left(t_{1}, \ldots, t_{n+1}\right)\right), m\right) \\
& =\left(m, u_{n}\left(t_{1}, \ldots, t_{n+1}\right), m\right) \\
& =\left(m, v_{n}\left(t_{1}, \ldots, t_{n+1}\right), m\right) \\
& =\left(m, b_{2,2}\left(w_{2,1}^{(n)}\left(t_{1}, \ldots, t_{n+1}\right), w_{2,2}^{(n)}\left(t_{1}, \ldots, t_{n+1}\right)\right), m\right) \\
& =b_{2,2}\left(\bar{w}_{2,1}^{(n+1)}, \bar{w}_{2,2}^{(n+1)}\right) \\
& =v_{n+1}\left(a_{1}, \ldots, a_{n+2}\right) .
\end{aligned}
$$

We conclude that $u_{n+1}=v_{n+1}$ holds in $B^{n+1}(T)$.

We now set out to show that $u_{n+1}=v_{n+1}$ does not hold in $B^{n+2}(T)$. By the induction hypothesis $u_{n}=v_{n}$ does not hold in $B^{n+1}(T)$. Thus, there exist $s_{1}, \ldots, s_{n+1} \in B^{n+1}(T)$ such that $u_{n}\left(s_{1}, \ldots, s_{n+1}\right) \neq v_{n}\left(s_{1}, \ldots, s_{n+1}\right)$. We now consider the elements

$$
a_{1}=\left(0, s_{1}, 1\right), \ldots, a_{n+1}=\left(0, s_{n+1}, 1\right), a_{n+2}=(n+1, e, 0)
$$

of $B^{n+2}(T)$, where $e$ denotes the identity element of $B^{n+1}(T)$. Let the element of $B^{n+2}(T)$ which results from $w_{j, 1}^{(n+1)}$ by substituting the variables $x_{1}, \ldots, x_{n+2}$ by $a_{1}, \ldots, a_{n+2}$, respectively, be denoted by $\bar{w}_{j, l}^{(n+1)}$ for all $j, l$. Then

$$
\begin{aligned}
\bar{w}_{n+1,1}^{(n+1)} & =\left(0, s_{1}, 0\right), \\
\vdots & \\
\bar{w}_{n+1, n+1}^{(n+1)} & =\left(0, s_{n+1}, 0\right), \\
\bar{w}_{n, 1}^{(n+1)} & =\left(0, w_{n, 1}^{(n)}\left(s_{1}, \ldots, s_{n+1}\right), 0\right), \\
\vdots & \\
\bar{w}_{n, n}^{(n+1)} & =\left(0, w_{n, n}^{(n)}\left(s_{1}, \ldots, s_{n+1}\right), 0\right),
\end{aligned}
$$


and using induction we obtain

Hence

$$
\bar{w}_{2, j}^{(n+1)}=\left(0, w_{2, j}^{(n)}\left(s_{1}, \ldots, s_{n+1}\right), 0\right), \quad j=1,2 .
$$

$$
\begin{aligned}
u_{n+1}\left(a_{1}, \ldots, a_{n+2}\right) & =a_{2,2}\left(\bar{w}_{2,1}^{(n+1)}, \bar{w}_{2,2}^{(n+1)}\right) \\
& =\left(0, a_{2,2}\left(w_{2,1}^{(n)}\left(s_{1}, \ldots, s_{n+1}, w_{2,2}^{(n)}\left(s_{1}, \ldots, s_{n+1}\right)\right), 0\right)\right. \\
& =\left(0, u_{n}\left(s_{1}, \ldots, s_{n+1}\right), 0\right) \\
& \neq\left(0, v_{n}\left(s_{1}, \ldots, s_{n+1}\right), 0\right) \\
& =\left(0, b_{2,2}\left(w_{2,1}^{(n)}\left(s_{1}, \ldots, s_{n+1}\right), w_{2,2}^{(n)}\left(s_{1}, \ldots, s_{n+1}\right)\right), 0\right) \\
& =b_{2,2}\left(\bar{w}_{2,1}^{(n+1)}, \bar{w}_{2,2}^{(n+1)}\right) \\
& =v_{n+1}\left(a_{1}, \ldots, a_{n+2}\right)
\end{aligned}
$$

and $u_{n+1}=v_{n+1}$ does not hold in $B^{(n+2)}(T)$.

\section{REFERENCES}

1. S. I. Adjan, Identities in special semigroups, Dokl. Akad. Nauk SSSR 43 (1962), 499-502 (in Russian).

2. T. S. Blyth and M. F. Janowitz, Residuation theory, (Pergamon Press, 1972).

3. A. H. Clifford and G. B. Preston, The algebraic theory of semigroups, Math. Surveys 7, (Amer. Math. Soc., Providence, Vol. I, 1961; Vol. II, 1967).

4. F. Pastijn and X. Yan, Completely regular semigroups in the variety generated by the bicyclic semigroup, Algebra Universalis, 30 (1993), 234-240.

5. F. Pastijn and $X$. Yan, Varieties of semigroups and varieties of completely regular semigroups closed for certain extensions, J. Algebra, 163 (1994), 777-794.

6. M. Petrich, Inverse semigroups, (Wiley, 1984).

7. J. G. Rosenstein, Linear orderings, (Academic Press, 1982).

Department of Mathematics, Statistics and Computer Science

MARQUETTE UNIVERSITY

MilwaukeE, WI 53233

USA 